\title{
Ascending pattern of sensory and motor symptoms due to metastatic epidural spinal cord compression
}

\author{
Kiyoshi Shikino ${ }^{1}$, Hiraku Funakoshi ${ }^{2}$, and Masatomi Ikusaka ${ }^{1}$ \\ ${ }^{1}$ Chiba University Hospital \\ ${ }^{2}$ Tokyo-bay Urayasu Ichikawa Medical Center
}

July 7,2020

\begin{abstract}
We should know that a common early pattern is radiculopathy at the level of compression along with an ascending pattern of sensory and motor symptoms in the distal lower extremities.
\end{abstract}

\section{Key Clinical Message}

MESCC may cause sensory and motor symptoms to appear first in the distal legs due to the specific lamination of the corticospinal and spinothalamic tracts (lower extremity fibers, most superficial and upper extremity fibers, the deepest).

An 84-year-old man experienced right-sided bundle-like chest pain 5 days before admission. Three days after the incident, he had symmetric ascending weakness and sensory loss in the distal legs. Neurological examination revealed the same conditions below the Th8 level. He had been receiving leuprorelin acetate and bicalutamide for prostate cancer. He exhibited exaggerated and Babinski reflexes in the legs. Spinal magnetic resonance imaging revealed that the epidural tissue process compressed the medullary cord in the ninth thoracic vertebra (Figure 1 and 2). He underwent posterior thoracic fusion surgery, and the symptoms immediately improved. Intraoperative pathological findings revealed a metastatic vertebral tumor, and metastatic epidural spinal cord compression (MESCC) was diagnosed.

MESCC occurs when cancer metastasizes to vertebrae or epidural space and causes compresses of spinal cord, which causes paraplegia if untreated. ${ }^{1}$ Owing to the specific lamination of the corticospinal and spinothalamic tracts (lower extremity fibers, most superficial and upper extremity fibers, the deepest), the thoracic compressive lesion may cause sensory and motor symptoms to appear first in the distal legs.

\section{Conflict of interest statement}

None of the authors have any conflicts of interest.

\section{Author's contribution}

All authors managed patient. KS wrote the draft. HF and MI revised this article.

\section{Reference}

Cole JS, Patchell RA. Metastatic epidural spinal cord compression. Lancet Neurol. 2008;7(5):459-66.

\section{Figure Legends}

Figure 1 
T2 weighted spinal magnetic resonance imaging (axial) revealed that the epidural tissue process compressed the medullary cord in the ninth thoracic vertebrae (Th9).

\section{Figure 2}

T2 weighted spinal magnetic resonance imaging (sagittal) revealed that the epidural tissue process compressed the medullary cord in the ninth thoracic vertebrae (Th9).

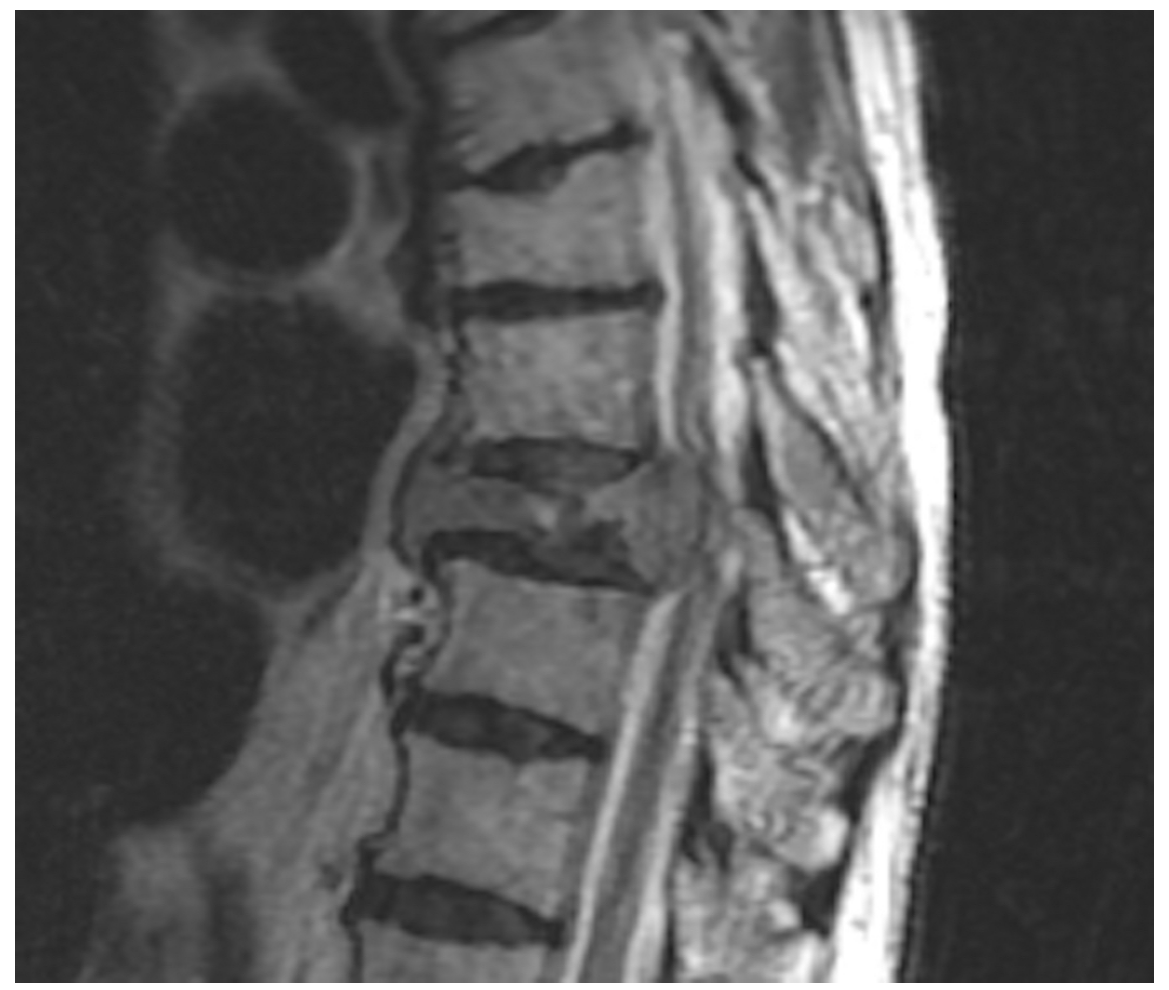




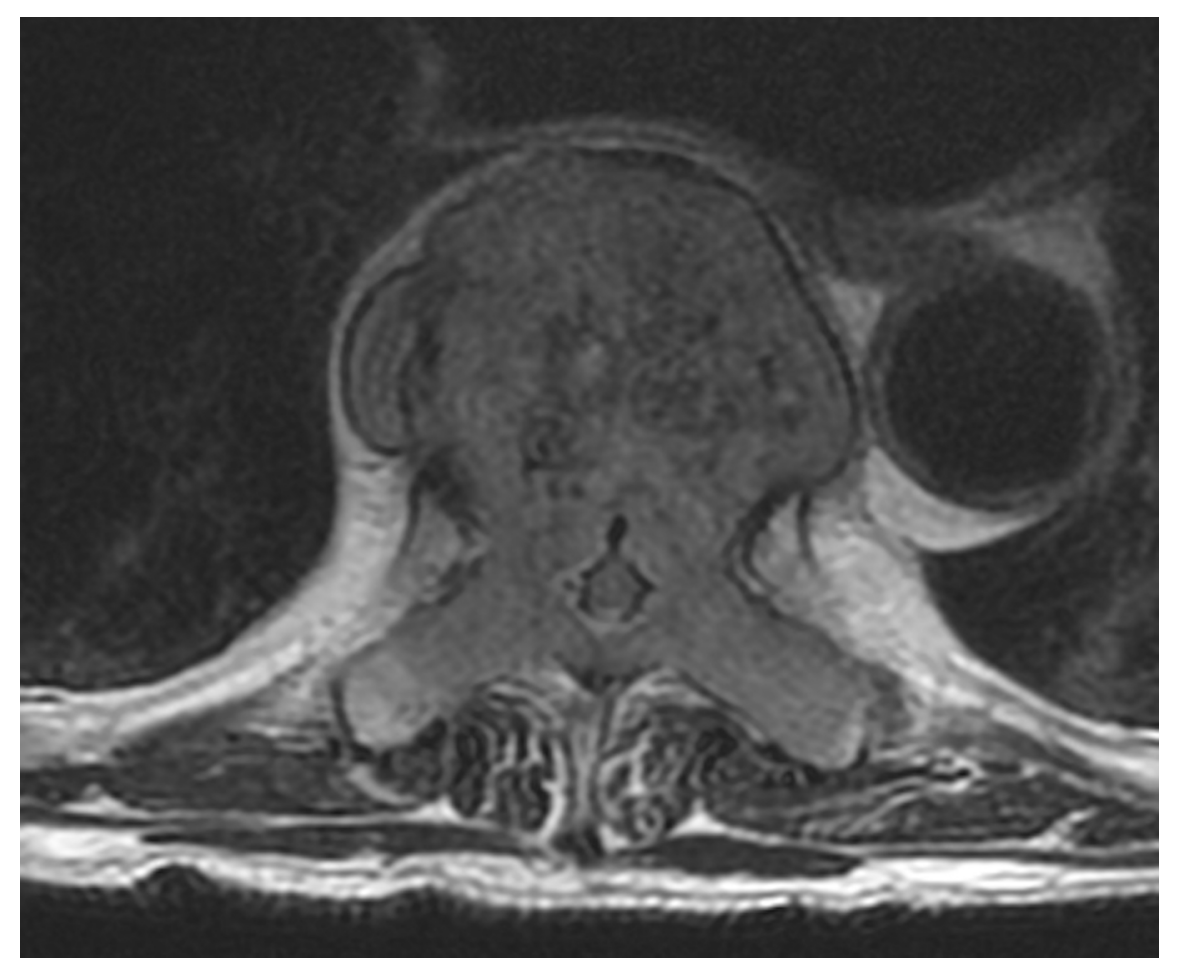

